\title{
Low-Voltage Cathodoluminescence as a High Spatial Resolution Technique for Nanostructure Characterization
}

\author{
E. Dupuy ${ }^{1,3}$, N.Pauc ${ }^{2}$, D.Morris ${ }^{3}$, M. Gendry ${ }^{3}$ and D. Drouin ${ }^{2}$
}

${ }^{1}$ Département de Physique, Université de Sherbrooke, J1K2R1, Sherbrooke, Québec, Canada

${ }^{2}$ Département de Génie Electrique et Génie Informatique, Université de Sherbrooke, J1K2R1, Sherbrooke, Québec, Canada

${ }^{3}$ Institut des Nanotechnologies de Lyon, UMR CNRS 5270, Ecole Centrale de Lyon, 69134, Ecully, France

Scanning cathodoluminescence (CL) microscopy and spectroscopy is a very powerful microanalysis technique for studying the optical and structural properties of bulk and low-dimensional semiconductor materials [1]. Growing interests for this technique are driven by its relatively high spatial resolution, its contactless character, and its ease of use without any special sample preparation. Moreover, this technique is generally non-destructive and can be combined with the normal imaging capabilities and analysis possibilities of the scanning electron microscope (SEM).

Investigation of single nanostructures requires a technique capable of sub-micron spatial resolution. Spatial resolution achievable in CL mode is a function of three parameters, which are the diameter of the exciting electron beam, the primary electrons scattering radius and the diffusion length of generated carriers. Combining a recent field emission electron source that provides a small and stable focused beam and a low-voltage acceleration source for the primary electrons, the interaction volume into which electron-hole pairs are created is reduced toward a quasi-point source. Under these conditions, the spatial resolution in CL is essentially limited by the diffusion length in the material. Therefore, the diffusion length of the excited species (carriers, excitons) can be directly measured. This approach has been successfully applied to map high density threading dislocations in GaN epilayer and extract the diffusion length from CL profile measured across a single dislocation core [2].

In this contribution, we present measurements of excess carrier diffusion lengths before capture by single self-assembled quantum dots (QDs) [3]. Measurements were performed on InAs/InP QDs emitting around $1.55 \mu \mathrm{m}$ by low-voltage cathodoluminescence in a SEM (Fig.1a). These semiconductor nanostructures are promising active materials for the realization of nanophotonic devices operating at telecom wavelengths, such as single-photon sources for quantum cryptography systems or entangled photon emitters for quantum computing. Investigating carrier transport in the vicinity of single QDs is thus relevant to optimize device performances.

Cathodoluminescence characterization was conducted using a Zeiss Supra 55 VP field emission gun SEM equipped with a cryogenic stage ( $5 \mathrm{~K}$ to room temperature) coupled to a Gatan MonoCL 2 setup with a cooled InGaAs photomultiplier tube. At low voltage $(1 \mathrm{kV})$, the SEM electron spot size is as small as about $3 \mathrm{~nm}$ and excess carriers are generated in sub-10-nm volume in InP providing a quasi-point source [4]. At $1 \mathrm{kV}$, we have spatially resolved individual QDs, which appear as correlated bright spots on CL micrographs (Fig.1c). A simple diffusion model adapted to our quantum heterostructure has been developed to extract the diffusion length. Fig.1b presents a schematic of the experiment. Excess carriers generated in sub-10-nm volume of the barrier are 
mainly captured by the wetting layer (WL) and diffuse through it before their final capture into a QD. Analysis of CL intensity profiles across single QDs provides a direct measurement of the ambipolar diffusion length in the InAs WL before capture into the dot (Fig.1d). Diffusion lengths are evaluated as a function of exciting beam intensity, temperature and sample doping.

References

[1] A. Gustafsson, J. of Microscopy, 224 (2006) (Pt 1) 72-78.

[2] N. Pauc, M. R. Phillips, V. Aimez, D. Drouin, Appl. Phys. Lett. 89 (2006), 161905.

[3] E. Dupuy, D. Morris, N. Pauc, V. Aimez, M. Gendry, and D. Drouin, Appl. Phys. Lett. 94 (2009) 032113.

[4] D. Drouin, A. R. Couture, D. Joly, X. Tastet, V. Aimez and R. Gauvin, Scanning 29 (2007) 92-101.

[5] This work was partly supported by Canadian grant funds (NSERC, FQRNT and Nano-Québec) and by the program Frontenac, which supports France/Québec exchange graduate student.

(a)
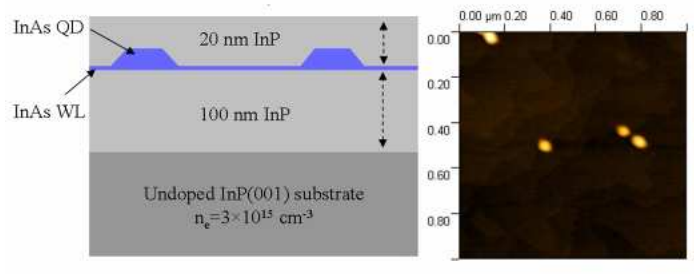

(c)

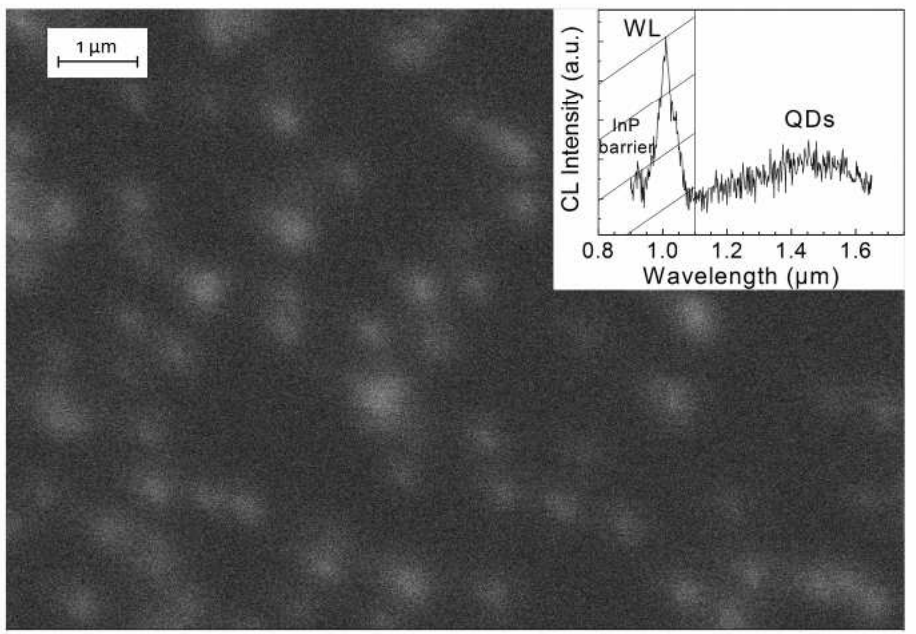

(b)

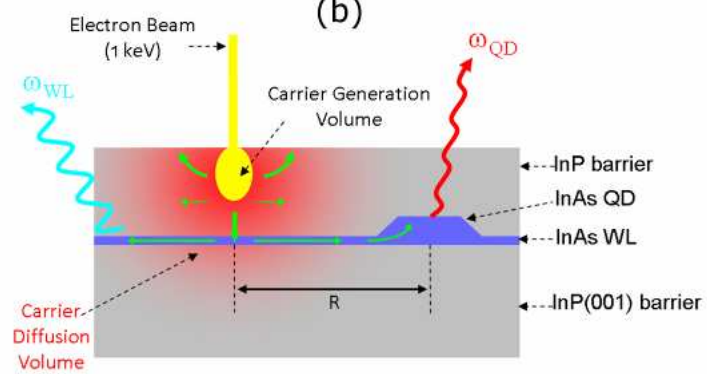

(d)

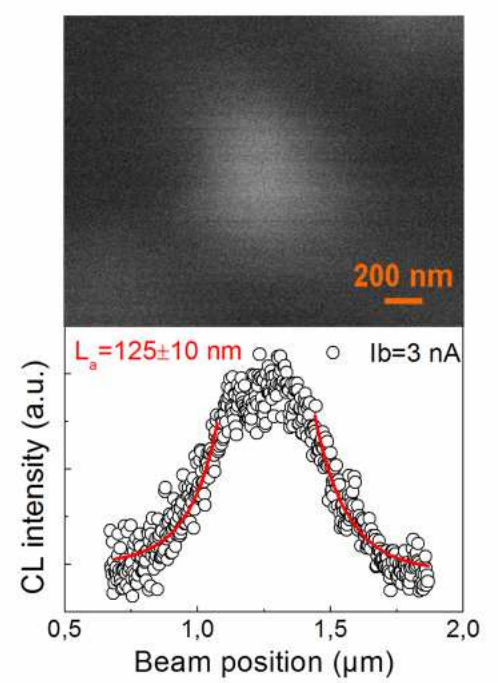

FIG. 1. (a) Schematic of the investigated sample and AFM images of low-density InAs/InP quantum dots (b) Schematic illustrating the measurement method of excess carrier diffusion lengths before their capture by individual quantum dots using low-voltage CL technique. (c) CL micrograph of the sample and his associated emission spectrum (inset). (d) Diffusion length measurement extracted from CL intensity profile taken across a single QD. 